Published in final edited form as:

Int J Drug Policy. 2014 May ; 25(3): 533-542. doi:10.1016/j.drugpo.2013.12.002.

\title{
Piloting a 'Spatial Isolation' Index: The Built Environment and Sexual and Drug Use Risks to Sex Workers
}

\author{
Kathleen N Deering ${ }^{1,2}$, Melanie Rusch ${ }^{1,3}$, Ofer Amram ${ }^{1,4}$, Jill Chettiar ${ }^{1,5}$, Paul Nguyen ${ }^{1}$, \\ Cindy X Feng ${ }^{6}$, and Kate Shannon ${ }^{1,2,5}$ \\ ${ }^{1}$ Gender and Sexual Health Initiative, BC Centre for Excellence in HIV/AIDS, St. Paul's Hospital, \\ Vancouver, Canada \\ ${ }^{2}$ Department of Medicine, Faculty of Medicine, University of British Columbia, Vancouver, Canada \\ ${ }^{3}$ Vancouver Island Health Authority, Victoria, Canada \\ ${ }^{4}$ Department of Geography, Faculty of Environment, Simon Fraser University, Burnaby, Canada \\ ${ }^{5}$ School of Population and Public Health, Faculty of Medicine, University of British Columbia, \\ Vancouver, Canada \\ ${ }^{6}$ School of Public Health, Faculty of Medicine, University of Saskatchewan, Saskatoon, Canada
}

\section{Abstract}

Background-Employing innovative mapping and spatial analyses of individual and neighborhood environment data, we examined the social, physical and structural features of overlapping street-based sex work and drug scenes and explored the utility of a 'spatial isolation index' in explaining exchanging sex for drugs and exchanging sex while high.

Methods-Analyses drew on baseline interview and geographic data (Jan/10-Oct/11) from a large prospective cohort of street and off-street sex workers (SWs) in Metropolitan Vancouver and external publically-available, neighborhood environment data. An index measuring 'spatial isolation' was developed from seven indicators measuring features of the built environment within $50 \mathrm{~m}$ buffers (e.g. industrial or commercial zoning, lighting) surrounding sex work environments. Bivariate and multivariable logistic regression was used to examine associations between the two outcomes (exchanged sex for drugs; exchanged sex while high) and the index, as well as each individual indicator.

\footnotetext{
(C) 2013 Elsevier B.V. All rights reserved.

Corresponding author/ reprints: Kathleen N Deering, PhD, Postdoctoral Research Fellow, Canadian Institutes of Health Research (Bisby Award) \& Michael Smith Foundation for Health Research, Division of AIDS, Faculty of Medicine, University of British Columbia; Analytic Research Coordinator, Gender and Sexual Health Initiative, British Columbia Centre for Excellence in HIV/ AIDS, 608 - 1081 Burrard Street, Vancouver, BC, Canada V6Z 1Y6, gshi@cfenet.ubc.ca, Ph: 604-682-2344 (ext 66268), Fx: 604-806-9044.

Publisher's Disclaimer: This is a PDF file of an unedited manuscript that has been accepted for publication. As a service to our customers we are providing this early version of the manuscript. The manuscript will undergo copyediting, typesetting, and review of the resulting proof before it is published in its final citable form. Please note that during the production process errors may be discovered which could affect the content, and all legal disclaimers that apply to the journal pertain.

Conflict of Interest Statement

We declare that we have no conflicts of interest including any financial, personal or other relationships with other people or organizations within three years of beginning the submitted work that could inappropriately influence, or be perceived to influence, the submitted work.
} 
Results-Of 510 SWs, 328 worked in street-based/outdoor environments (e.g. streets, parks, alleys) and were included in the analyses. In multivariable analysis, increased spatial isolation surrounding street-based/outdoor SWs' main places of servicing clients as measured with the index was significantly associated with exchanging sex for drugs. Exchanging sex for drugs was also significantly positively associated with an indicator of the built environment suggesting greater spatial isolation (increased percent of parks) and negatively associated with those suggesting decreased spatial isolation (increased percent commercial areas, increased count of lighting, increased building footprint). Exchanging sex while high was negatively associated with increased percent of commercial zones but this association was removed when adjusting for police harassment.

Conclusions-The results from our exploratory study highlight how built environment shapes risks within overlapping street-based sex work and drug scenes through the development of a novel index comprised of multiple indicators of the built environment available through publicly available data, This study informs the important role that spatially-oriented responses, such as safer-environment interventions, and structural responses, such as decriminalization of sex work can play in improving the health, safety and well-being of SWs.

\section{Keywords}

sex workers; sex work; drug use; HIV risk; spatial analysis

\section{INTRODUCTION}

Increasing theoretical, qualitative and social epidemiological work has elucidated the important role of place, including the dynamic interplay between social context and physical and structural environments, on influencing health risks experienced by vulnerable and marginalized populations, including sex workers (SWs) and people who use drugs (Tempalski \& McQuie, 2009). Rhodes' 'risk environment framework' has been particularly useful in re-conceptualizing drug use harms, including drug-related harms, as being produced by social situations and places rather than solely by individual 'risk behaviours', with the 'risk environment' defined as the "space...in which a variety of factors interact to increase the chances of drug-related harm" (Rhodes, 2002). Related research has advocated for conceptual and analytic methods that can account for the effects of social and physical factors operating on multiple and interrelated levels, including on the level of populations (macro), community (meso) and individual (micro) on HIV risk (Aral, Padian, \& Holmes, 2005; Diez Roux \& Aiello, 2005; Rhodes, 2009). Further work by Blanchard and Aral conceptualizes sex work as a complex system, whereby the overlap of social context and physical and structural environments of sex work interact to produce HIV risk; for example, in settings where sex work is highly criminalized and stigmatized, sex work environments remain largely hidden and isolated, highly mobile and controlled by pimps or brokers who connect SWs with clients (Blanchard \& Aral, 2010).

In settings where sex work and drug use markets overlap, place- and gender-based dual drug use and sexual risks for women may be exacerbated. The male-dominated nature of street culture within such settings and the gendered dynamics of public spaces, wherein power relations favour male drug use and sexual partners, shape the negotiation of sexual practices 
(Bourgois, Prince, \& Moss, 2004; Rhodes, et al., 2012; Shannon, et al., 2008a). In such settings, sexual HIV transmission is thought to have been driven by the advent and increase in crack use since the early 1990s, the demands of which required women in particular to trade sex for drugs and negatively affected the amount of money for sex acts and power in negotiations with clients (Maher, 1997; Maher \& Curtis, 1992; Maher, Dunlap, Johnson, \& Hamid, 1996; Shannon, et al., 2008a). Limited research suggests that sex-for-drug exchanges are riskier than exchanging sex for money and have been associated with crack use and unprotected sex and sex with a drug user (Kwiatkowski \& Booth, 2000). SWs who exchange sex for drugs or exchange sex while high are also less likely to be able to negotiate terms with clients (e.g., safer sex), more likely to engage in riskier sexual practices (e.g., sex without condoms, anal sex) in exchange for immediate drugs and less able to control the drug preparation process (e.g., assess drug quality/safety, share drug use equipment as second user) (Maher \& Curtis, 1992; Maher, et al., 1996; Shannon, Kerr, Bright, Gibson, \& Tyndall, 2008b).

Key features of physical locations of sex work environments can play an important role in gender-based dual drug use and sexual risks to SWs, particularly in settings where sex work is criminalized. The geographic concentration of sex work in more hidden and isolated spaces is often a "socially acceptable" strategy with the goal of removing the visible presence of sex work from the public eye (e.g., from streets, windows). Removing sex work from public spaces can happen explicitly through regulation (e.g. municipal zoning restrictions on working in specific areas of a city) or through the creation of formal tolerance zones (e.g. 'red light districts') (Hubbard \& Whowell, 2008; Lowman, 1992) or 'defacto tolerance zones' due to local policing and fear of police harassment and arrest (Hubbard, 1998). Spatial isolation of SWs, including through policing practices related to enforcement of sex work laws, has been associated with increased health harms to SWs, including gender-based violence, risky sexual or drug-related behaviours (e.g., unsafe sex; sharing drug use equipment) and lack of access to health services (Lazarus, Chettiar, Deering, Nabess, \& Shannon, 2011; Rhodes, Simic, Baros, Platt, \& Zikic, 2008; Shannon, et al., 2008a; Shannon, et al., 2009).

This qualitative and social epidemiological research has been integral in identifying the importance of features of place, including spatial isolation, on negative health risks among SWs; however this research has largely relied on individual self-reported experiences and descriptions of the individual's environment. Critical work within the drug use and sexual health literature has examined the effects of social context and physical and structural environments on health via aggregated or cumulative effects through indicators that measure features of the built environment. The term 'built environment', has broad uses and applications, and in our paper refers to features of human-made spaces, places or surroundings in which human activity takes place. For example, the relationship between spatial access to sterile syringes, policing of drug use (arrests) and the use of safe drug use equipment has been assessed (Cooper, et al., 2012a; Cooper, et al., 2012b). An index measuring the cumulative effects of physical disorder within neighbourhoods (e.g., structural damage to homes; streets with trash, abandoned cars, graffiti; physical problems and building code violations in high schools), the 'Broken Window Index', was examined for its influence on neighborhood gonorrhea rates in New Orleans (Cohen, et al., 2000). 
Despite important contributions of this work to understanding the effects of features of place, including within the built environment among marginalized and street-involved populations, to date, there has been little to no cross-dialogue between built environment research and spatial research of health inequities at the population level and qualitative and social epidemiological research of social and health harms at the individual level (e.g. cohort data, qualitative in-depth interviews), particularly within sex work research.

Through employing innovative mapping with a large cohort of SWs and spatial analyses of both individual data and neighbourhood environment data, our exploratory study aimed to address these gaps in research by examining the social, physical and structural features in overlapping street-based sex work and drug scenes. Guided by existing theoretical, qualitative and social epidemiological research, we explored the utility of a spatial isolation index of SWs' built environment, and the relationship between this index and two dual drug use and sexual risk outcomes: exchanging sex while high and exchanging sex for drugs. We also examined the individual effects of built environment indicators that were used to develop the index on the outcomes. In addition, given substantial evidence of the influence of policing practices on influencing sex work activities as well as the spaces where sex work is practiced within settings where sex work is criminalized, we aimed to explore the potential confounding effect of police harassment on the relationship between built environment indicators and our two outcomes. This research is situated in Vancouver, Canada, a setting with criminalized policies toward both sex work (i.e., communicating/ soliciting for the purposes of prostitution; owning and operating a brothel/bawdy house; and living off the avails of prostitution) and drug use.

\section{METHODS}

\section{Survey design and sample}

Beginning in January 2010, youth and adult women (14 years+) were enrolled in a longitudinal cohort known as 'An Evaluation of Sex Worker's Health Access' ('AESHA'). This study is based on substantial community collaborations (e.g., sex work agencies and service providers) existing since 2005, and is monitored by a Community Advisory Board with representatives from 15+ agencies. Using time-location sampling,(Stueve, O'Donnell, Duran, San Doval, \& Blome, 2001) women who exchanged sex for money within the last 30 days (SWs) were recruited through outreach to outdoor sex work locations (i.e. streets, alleys), indoor sex work venues (i.e. massage parlours, micro-brothels, and in-call locations) and independent/self-advertising SWs (e.g. online, newspapers) in Metropolitan Vancouver. Our eligibility is inclusive of transgender individuals (male-to-female, MTF) who identify as women, based on our previous work (Shannon, 2007) and community guidance, as MTF transgender individuals work in similar spaces as the female SW population, and access the same services as the female SWs (directed toward self-identifying women, transgender inclusive). Interviews were conducted in places where women felt comfortable (i.e., three office site locations across Vancouver; within indoor sex work venues). As executed previously, outdoor sex work 'strolls' and indoor venues were identified through a participatory mapping exercise conducted with current/former SWs (Shannon, 2007), and continuously updated by the outreach team. The study holds ethical approval through 
Providence Health Care/University of British Columbia Research Ethics Board. All participants receive an honorarium of \$40CAD at each bi-annual visit for their time, expertise and travel.

\section{Questionnaires and measures}

Following informed consent, at baseline and each semi-annual follow-up visit, participants completed questionnaires by trained interviewers (both SW and non-SW interviewers) that elicited responses relating to socio-demographics, sex work patterns/client experiences, work environments, occupational violence and interactions with policing, characteristics of non-commercial or regular partnerships, violence and trauma, and drug use. Participants also completed a nurse-administered questionnaire that elicited responses relating to overall physical, mental and emotional health, sexual and reproductive health and HIV testing and treatment. As part of the nursing visit, SWs were also provided with extensive pre/and posttest counseling, testing for HIV, Hepatitis C Virus and sexually transmitted infections, and referral for care and support services. Treatment was provided for symptomatic STI infections by an on-site nurse, and free serology and Papanicolaou testing were also available for those in need, regardless of study enrollment.

\section{Study sample}

Our study sample included SWs who solicited for or serviced clients primarily in streetbased/ outdoor settings. We considered only baseline data.

\section{Outcomes}

Based on a priori interest in examining overlapping drug use and sexual risks, we included two sexual risk outcomes in this analysis, measured in the last six months: (1) exchanged sexual services directed for drugs ('yes'=always, usually, sometimes, occasionally; versus 'no'=never); and (2) exchanged sexual services while high ('yes'=always, usually, sometimes, occasionally; versus 'no'=never).

\section{Potential confounders}

We considered the following potential confounders as measured once at baseline: age; reporting being a sexual minority (lesbian, gay, bisexual, transgender, transsexual, two-spirit versus heterosexual and non-transgender); Indigenous/Aboriginal ancestry (including First Nations and Métis, Inuit status); migrant/new immigrant status (born outside Canada); and age at first sex work; as well as time-varying confounders updated in the last six months: homeless; had a manager; non-injection drug use; injection drug use; and numbers of clients per week; and experienced police harassment without arrest (i.e., told to move on, threatened with arrest/detainment/fine, searched, followed, picked up and driven elsewhere to work, verbally harassed, detained, delayed/held against will without arrest, physically assaulted, drugs/drug use equipment taken, other property taken, propositioned to exchange sex, coerced into providing sexual favours). 


\section{Selection of built environment indicators}

We used conceptual and analytic methods in the exploratory development of a 'spatial isolation index' within street-based/outdoor sex work environments, using seven variables measuring different aspects of the built environment (Table 1). In the current study, the 'spatial isolation index' and the term 'spatial isolation' in general is intended to reflect how sex work in our setting has been geographically concentrated in more hidden and isolated spaces, away from the public eye (e.g., busy streets, residential areas) - research has suggested that these spaces are moreover often further away from health resources (e.g., harm reduction and safer sex equipment) and safety resources (e.g., groups of people, other SWs, drop-in spaces, street-lights/busier commercial settings) (Shannon, et al., 2008a; Shannon, et al., 2009). To derive the seven individual built environment indicators for use in development of an index, we used a combination of AESHA survey data and seven variables measuring features of the built environment collected from publicly available data from the City of Vancouver ("City of Vancouver Open Data Catalogue," 2013) and DTMI Spatial ("DMTI Spatial Data and Services, " 2013). Little research is available on community-level indicators of built environment and their relationships with health harms to street-based SWs. As this study was exploratory in nature, we developed indicators that were suggested by research to be important in shaping risk environments to SWs in street-based/outdoor settings (Shannon, et al., 2008a; Shannon, et al., 2008c). From the AESHA data, we used two variables measuring key spatial aspects of SWs' work environments in the development of these indicators: locations of SWs' main places of solicitation and main places where services were provided to clients, in the last six months. Locations were provided by participants in the form of addresses or cross-streets and geocoded (i.e., assigned latitude and longitude). Using ArcGIS 10.1 ("ArcGIS Desktop," 2011), around each location, spatial buffers were created. Effectively, 'buffers' are equivalent to a circle drawn around each location as provided by participants. Each circle had an assigned radius of $50 \mathrm{~m}$, as we were interested in assessing the effects of built environment within a relatively near proximity to primary sex work environments. These effects were perceived to be likely to have the largest effect on SWs' HIV risk. In line with previous work by our team (Rusch, et al., 2010; Shannon, et al., 2008c), confidentiality of participants' individual responses was ensured as only aggregate measures of sex work spaces were used in analysis. Neighbourhood environment variables drawn from four different groups of external/publicly available data were further used in the development of the index: road, lighting, building footprint and land use ("City of Vancouver Open Data Catalogue," 2013; "DMTI Spatial Data and Services," 2013). These variables included, within each buffer, the: (1) sum of the length of major roads; (2) sum of the length of alley roads; (3) percentage of commercial land use; (4) percentage of industrial land use; (5) percentage of parks; (6) number of light posts; and (7) percentage of the building footprint, or total built environment (i.e., coverage of land by commercial buildings) (Table 1, Figure 1).

\section{Development of an index for $50 \mathrm{~m}$ buffer}

We drew on other studies that have developed indices of the built environment using multiple indicators of the built environment (e.g., neighbourhood disorder; street speed limits, volume of cars, and street connectivity, walkability, land use, dwelling density) 
(Badland, et al., 2009; Cohen, et al., 2000; Kroeger, Messer, Edwards, \& Miranda, 2012; McGinn, Evenson, Herring, Huston, \& Rodriguez, 2007).

Step 1: Conceptually, we hypothesized that greater major road length, greater percent of commercial land use and greater number of light posts was associated with decreased spatial isolation (as defined above), while greater length of alley roads, industrial land use and parks was associated with increased spatial isolation. Greater percent of building footprint could be associated with increased spatial isolation or decreased spatial isolation (e.g., building footprint could represent commercial buildings or industrial/abandoned, so the impact of the overall footprint size would depend on type of building. These indicators were conceptualized as described based on the previous work by Shannon et al (2008a; 2008c). These relationships were also validated from extensive discussion with project staff and community members who are experts in understanding the experiences of the population under study.

Step 2: For the seven items, we used simple correlations to determine the directions of items and how they were related to provide evidence to support out hypotheses. Results from Table 2a (built environment indicators for place of solicitation) support our hypothesis, suggesting that major road length, percent commercial land use and number of lighting posts within 50m buffers surrounding SWs' main places of solicitation were positively correlated, while negatively correlated with alley road length, percent industrial land use and percent parks within 50m buffers. Interestingly, alley road length, percent industrial land use and percent parks within $50 \mathrm{~m}$ buffers were also negatively correlated with each other. Building footprint was strongly negatively correlated with major road length $(\mathrm{p}<0.001)$ and positively correlated with percent industrial land use $(\mathrm{p}<0.001)$, suggesting that it could be more strongly associated with a measure of isolation. Results from correlations for built environment indicators derived for 50m buffers surrounding SWs' places of servicing clients were identical to those derived for $50 \mathrm{~m}$ buffers surrounding places of solicitation (Table $2 \mathrm{~b}$ ).

Step 3: Based on our hypotheses and empirical analysis in Step 1 and Step 2, we then developed an index or summary measure of the seven built environment indicators to measure 'spatial isolation' within street-based/outdoor sex work environments. Each indicator was divided into deciles, with each of the ten deciles scored 1-10 or 10-1, depending on the hypothesized direction of association with spatial isolation. We assigned scores based on percentiles because indicators were measured with different units (i.e., percent of industrial zoning versus number of light posts), to make indicators comparable. We validated the analysis using the index derived using quintiles (scored 1-5 or 5-1) and quartiles (scored 1-4 or 4-1).

\section{Analysis}

Bivariate and multivariable logistic regression was performed for the relationship between the index and the two outcomes. Odds ratios were interpreted as follows: for each one-unit increase in the index, the odds of the outcome increased/decreased by X\%. We also performed bivariate and multivariable logistic regression for the relationship between each individual built environment indicator and the two outcomes. Individual built environment indicators were standardized for comparability, as we were interested in reporting the 
direction of association and its associated statistical significance rather than interpreting odds ratios. For completeness, odds ratios were reported for multivariable associations and interpreted as follows: for each one-unit increase in the standardized built environment indicator, the odds of the outcome increased/decreased by $\mathrm{X} \%$. We considered multivariable relationships between individual built environment variables and the outcome when each pair of variables was statistically significantly associated at a $\mathrm{p}<0.10$-level, as this is pilot and exploratory analysis and we were interested in examining a broader number of comparisons. We hypothesized that increased spatial isolation (as measured by our index, as well as by individual built environment indicators that comprise the index - described below) will be associated with higher odds of exchanging sex while high and exchanging sex for drugs. As such, for each regression model, we used a confounder model approach using the methods of Maldonado and Greenland (Maldonado \& Greenland, 1993). As previously (Lima, et al., 2008; Maldonado \& Greenland, 1993), potential confounders were selected for inclusion in the final models using a backward selection approach, which considered the magnitude of change in the coefficient of the exposure variable. Starting with a fixed model, which considered all available variables, potential confounders were dropped one at a time, using the relative change in the coefficient for the variable related to the exposure variable as a criterion, until the maximum change from the full model exceeded 5\%. All analyses were performed using SAS software version 9.3 ("SAS Version 9.3," 2012).

\section{RESULTS}

\section{Characteristics of street-based/outdoor SWs}

Of 510 SWs within the AESHA cohort, 328 solicited for clients in primarily street-based/ outdoor environments (e.g. streets, parks, alleys) and were included in the analyses, with a median age of 34 years (Interquartile range: 29-43 years) and 45.5\% (149) of Aboriginal ancestry (First Nations, Metis, Inuit), 18.9\% (62) reporting being a migrant/new immigrant and $23.5 \%$ (77) reporting being a sexual minority. Overall, $77.1 \%$ and $45.7 \%$ used noninjection and injection drugs in the last six months, respectively. In the last six months, $70.7 \%$ (232) reported exchanging sex while high, while 32.6\% (107) reported exchanging sex for drugs and $43.3 \%$ reported police harassment without arrest.

\section{Dual sexual and drug use-related risk and spatial isolation index}

Table 3 presents bivariate associations between three derivations of the index (deciles, quintiles and quartiles) for place of soliciting and place of servicing clients and the outcomes measuring dual drug use and sexual risk (exchanging sex while high and exchanging sex for drugs). Increased spatial isolation of SWs, as measured by our index, was strongly positively and significantly associated with both outcomes. These results were consistent across the index derived using deciles, quintiles and quartiles, and for buffers surrounding SWs' main places of solicitation and of servicing clients.

Table 4 presents multivariable associations between three derivations of the index (deciles, quintiles and quartiles) for place of soliciting and place of servicing clients and exchanging sex while high and exchanging sex for drugs. In multivariable analysis, after adjusting for 
key confounders, exchanging sex for drugs remained positively and significantly associated with the index measuring spatial isolation of SWs, for the index derived for SWs' main place of servicing clients and for deciles (AOR: 1.03, 95\%CIs: 1.00-1.05), quintiles (AOR: 1.06, 95\%CIs: $1.01-1.10$ ) and quartiles (AOR: 1.09, 95\%CIs: 1.02-1.16).

\section{Dual sexual and drug use-related risk, police harassment and built environment indicators}

In bivariate analysis, for individual built environment indicators, as derived for $50 \mathrm{~m}$ buffer around SWs' main place of solicitation, exchanging sex while high was significantly associated with the following indicators on a $\mathrm{p}<0.10$-level: length of major roads $(0.024)$; percent commercial zoning $(\mathrm{P}=0.006)$; percent industrial zoning $(\mathrm{P}=0.048)$; and percent parks $(\mathrm{P}=0.077)$. Exchanging sex for drugs was significantly associated with: length of major roads $(\mathrm{P}=0.057)$; and percent parks $(\mathrm{P}=0.008)$. In bivariate analysis, for individual built environment indicators, as derived for $50 \mathrm{~m}$ buffer around SWs' main place of servicing, exchanging sex while high was significantly associated with the following indicators on a $\mathrm{p}<0.10$-level: length of alleys $(<0.001)$; percent commercial zoning $(0.063)$; percent industrial zoning (0.026); percent parks (0.074). Exchanging sex for drugs was significantly associated with: length of major roads $(0.071)$; percent commercial zoning (0.005); percent parks (0.001); number of light posts (0.022); and percent building footprint $(0.033)$.

Tables 5a and 6a present multivariable relationships between individual built environment indicators and these two outcomes, developed for buffers surrounding places of solicitation and servicing, respectively. Tables $5 \mathrm{~b}$ and $6 \mathrm{~b}$ presents the same analysis, with the multivariable models for the two outcomes adjusted for police harassment. For the buffers developed surrounding places of solicitation, in multivariable analysis, reduced odds of exchanging sex while high (AOR: $0.76,95 \%$ CIs: $0.59-0.99$ ) were significantly associated with increased percent of commercial areas. Elevated odds of exchanging sex for drugs were associated with increased percent of parks (AOR: 1.36, 05\% CIs: 1.06-1.75) (Table 5a). For the models where police harassment was identified as a key confounder, after adjusting for police harassment, the significant association between exchanging sex while high and increased percent of commercial areas was removed, but the association between exchanging sex for drugs and increased percent of parks remained (AOR: 1.38, 95\%CIs: 1.07-1.78) (Table 5b).

For the buffers developed surrounding places of servicing, in multivariable analysis, reduced odds of exchanging sex while high (AOR: $0.69,95 \%$ CIs: $0.53-0.89$ ) were significantly associated with increased length of alleys. Reduced odds of exchanging sex for drugs were associated with increased percent of commercial zoning (AOR: 0.72, 95\%CIs: 0.57-0.92), increased count of lighting (AOR: 0.73, 95\%CIs: 0.56-0.93) and increased percent of building footprint (AOR: 0.75, 95\%CIs: 0.60-0.95). Elevated odds of exchanging sex for drugs were significantly associated with increased percent of parks (AOR: 1.58, 95\%CIs: 1.14-2.18) (Table 6a). For the relationships where police harassment was identified as a key confounder, the association between exchanging sex for drugs and increased percent of parks (AOR: 0.74, 95\%CIs: 0.58-0.93) and increased building footprint (AOR: 0.76, 95\%CIs: 0.60-0.97) remained (Table 6b). 


\section{CONCLUSIONS}

Novel social epidemiological and spatial methods that integrate neighbourhood environment and individual level data can help us understand how social, physical and structure features of place shape potential health harms for marginalized populations. In particular, our exploratory research suggests that a 'spatial isolation' index within street-based sex work scenes can help illuminate how physical and structural features of the built environment may increase dual drug use and sexual risks, and inform policy and interventions.

We hypothesized that each built environment indicator represented a measure of 'spatial isolation' from some service or resource that is important in contributing toward reducing negative health outcomes among SWs (e.g., health services, safer sex and drug equipment resources and personal safety resources), and that increased spatial isolation would be associated with increased vulnerability to health harms as represented by increased sex-fordrug exchanges and exchanging sex while high. In developing our index, we were unable to validate externally that our index measured what we intended it to and were limited by availability of variables in external databases. However, our results were in the direction we would expect based on previous research and in line with our hypotheses. Increased spatial isolation surrounding street-based/outdoor SWs' main places of servicing clients, as measured with an index that took into account the effects of multiple built environment indicators and was intended to provide an overall measure of SWs' spatial isolation from resources and services, was significantly associated with exchanging sex for drugs. When assessed individually in multivariable analysis for built environment indicators surrounding SWs' main places of solicitation and servicing, elevated odds of sex-for-drug exchanges were significantly associated with an indicator of the built environment hypothesized to be associated with greater spatial isolation (increased percent of parks). Moreover, reduced odds of sex-for-drug exchanges were significantly associated with built environment indicators surrounding SWs' main places of servicing hypothesized to be associated with decreased spatial isolation (increased percent commercial areas, increased count of lighting, increased building footprint).

Given evidence highlighting the connections between police harassment of SWs and spatial isolation of SWs to places further away from public spaces and commercial areas, as well as away from places where health and harm reduction services are accessed (Hubbard, 1998; Lowman, 1992, 2000) (Kerr \& Wood, 2005; Shannon, et al., 2008a; Shannon, et al., 2008c), our index can be viewed as a potential marker for such policing practices, in addition to its more direct interpretation as an overall representation of isolation from resources and services. This observation is supported by results (not shown) suggesting bivariate relationships between reduced police harassment without arrest and increased: percent commercial zoning ( $\mathrm{P}=0.001)$; number of light posts $(\mathrm{P}=0.032)$; and percent building footprint $(\mathrm{P}=0.089)$ (i.e., markers of spatial isolation). Heavier police presence has been associated with increased risk for transmission of blood-borne or sexually transmitted infections to SWs and drug users through a number of spatial pathways; for example, heavier arrest rates have negatively affected the association between increased spatial access to sterile syringes and the use of safe drug use equipment (Cooper, et al., 2012a; Cooper, et al., 2012b); increased police presence and previous arrests/harassment by police have been 
associated with rushed negotiations with clients or rushed and unsafe drug use, injecting and doing sex work in unsafe spaces and having safer sex and drug use equipment confiscated (Kerr \& Wood, 2005; Shannon, et al., 2008a; Small, Kerr, Charette, Schechter, \& Spittal, 2006); and being able to access safer indoor drug use spaces has alleviated the pressures of policing and violence in public drug use spaces and facilitated use of safer drug use equipment (Fairbairn, Small, Shannon, Wood, \& Kerr, 2008). Such research helps explain our association between increased spatial isolation as measured by our index and exchanging sex for drugs. Moreover, our results showed that without adjusting for police harassment, working in areas with increased percent of commercial zones was associated with reduced risk for exchanging sex while high; however in multivariable analysis, this association was removed when adjusting for police harassment, which was identified as a key confounder in this relationship and had the strongest association with exchanging sex while high (5.7-fold elevated odds) relative to other confounders.

Spatial and place-based research on risk environments are critical to health policy as study results "can locate precisely places of potential risk environments, social vulnerability and where structural interventions are urgently needed" (Tempalski \& McQuie, 2009). Given that our results indicate that SWs with dual drug use and sexual risks may be at heightened risk of spatial isolation that could enhance their risks our study suggests a number of policy implications that are spatially oriented. Research suggests that development and scale-up of safer environment interventions (e.g., mobile outreach (Deering, et al., 2011; Janssen, Gibson, Bowen, Spittal, \& Petersen, 2009)) that modify the physical environments of streetbased/outdoor SWs can be instrumental in reducing sexual and drug-related harms. In addition, in line with global calls (Ahmed, Kaplan, Symington, \& Kismodi, 2011; Nosyk \& Wood, 2012; Shannon, 2010; Shannon \& Csete, 2010; Wood, McKinnon, Strang, \& Kendall, 2012),, there is strong evidence and global policy support (e.g.,: WHO/UN guidelines, Global Commission for HIV and the Law) for decriminalization of communicating for purposes of sex work in public/outdoor spaces ('communicating code') to ensure access to safer indoor spaces for sex work with supportive policies (e.g., occupational health and safety standards, supportive and safer policies toward drug use) that support SWs ability to better control sexual transactions and reduce health harms to SWs (Shahmanesh, Patel, Mabey, \& Cowan, 2008)).(Ghose, Swendeman, \& George, 2011; Kerrigan, et al., 2003; Krusi, et al., 2012; Withers, Dornig, \& Morisky, 2007). The shift away from policing tactics toward street-based/outdoor sex work that result in sex work spaces being moved into areas where there is limited access to health and safety resources could increase dialogue between SWs and police and contribute to the development of street-based/public sex work spaces that satisfy the needs of SWs along with community residents. In such cases, it may be possible that police presence could have a positive impact on SWs' sexual and drug-related risk. Safer-environment interventions would also be facilitated in a decriminalized environment, but are critical in a criminalized environment, particularly when places of sex work solicitation and servicing are known to be in areas where SWs are less able to access health and safety resources.

This study had a number of strengths as well as limitations that should be taken into account when interpreting results. Since sampling frames are difficult to construct for hidden populations, the sample was not randomly generated and may not be representative of all 
street-based/ outdoor SWs in ours or other settings. To address this, we recruited participants through systematic time-location sampling and targeted outreach to sex work strolls and indoor locations (Stueve, et al., 2001), considered the best method of recruitment for mobile/ hidden populations and therefore helping attract a representative sample. The study design is cross-sectional in nature and thus cannot determine causal relationships between spatial isolation and increased sexual and drug use risks to sex workers. As with all self-report data, responses may be subject to recall or social desirability bias. To counteract these potential biases, we had extensively trained interviewers with experience with the sample population, and interviews were conducted in spaces where women were comfortable (i.e., indoor work places), facilitating accurate responses. As discussed above, there is growing research suggesting that spatial isolation (e.g., through policing practices) can result in sex workers experiencing heightened health risks through sexual and drug injection routes. Few prospective studies with sex workers have been conducted, however, limiting the strength of conclusions regarding these relationships; our study points to areas where such research is needed.

There are some specific limitations with respect to the development of the index. For example, one limitation of the indicators used to develop our index is related to temporality. The relative 'spatial isolation' of a particular feature of the built environment (e.g., percentage of parks, number of streetlights) may differ according to the time of day/night. For example, the impact of reduced number of streetlights will be different for sex workers who work daytime hours than those who work nighttime hours. We were unable to account for the exact times at which SWs work. In our study setting, however, where sex work is criminalized, the majority of street-based sex work occurs during nighttime hours in order to avoid enforcement of sex work laws governing public solicitation; during nighttime hours, the impacts of various features of the built environment are likely to be relatively consistent. Our index measuring spatial isolation was moreover derived using external environmental data on built environment rather than individual-level self-report data. The intent of using data external to individuals was to remove subjective interpretations of environments on risk, which has been shown to have poor agreement with 'objective' indicators of built environments (McGinn, et al., 2007).

In summary, the results from our exploratory study highlight how built environment shapes risks within overlapping street-based sex work and drug scenes through the development of a novel index comprised of multiple indicators of the built environment available through publicly available data. The process through which this index was developed and the index itself can be used as a key starting point from which to build on to better understand the relationship between built environment and drug use and sexual risk to SWs in Vancouver and other settings. Indicators that are setting-specific should be chosen for specific settings. We have demonstrated that publicly available spatial data can be very useful in providing an external viewpoint of the relationships between space, place and risk to sex workers, and provides unique insights that cannot be gained from self-report data alone. However, the complex and multi-level nature of these relationships would benefit from further developments to the index, including incorporating data from multiple sources and methodologies, such as ethnographic and qualitative assessments, quantitative survey data 
alongside external data. This study informs the important role that spatially-oriented and structural responses can play in improving the health, safety and well-being of SWs.

\section{Acknowledgments}

We thank all those who contributed their time and expertise to this project, including participants, partner agencies and the AESHA Community Advisory Board. We wish to acknowledge Peter Vann, Gina Willis, Annick Simo, Ofer Amram, Paul Nguyen, Sabina Dobrer, Jill Chettiar, Jennifer Morris, Alex Scot and Kathleen Deering for their research and administrative support. This research was supported by operating grants from the US National Institutes of Health (R01DA028648) and Canadian Institutes of Health Research (HHP-98835). KND is supported by Postdoctoral Fellowships from the Canadian Institutes of Health Research (Bisby Award) and the Michael Smith Foundation for Health Research. KS is supported by US National Institutes of Health (R01DA028648), Canadian Institutes of Health Research and Michael Smith Foundation for Health Research.

\section{REFERENCES}

Ahmed A, Kaplan M, Symington A, Kismodi E. Criminalising consensual sexual behaviour in the context of HIV: Consequences, evidence, and leadership. Global Public Health. 2011; 6:S357S369. [PubMed: 22050481]

Aral SO, Padian NS, Holmes KK. Advances in multilevel approaches to understanding the epidemiology and prevention of sexually transmitted infections and HIV: an overview. Journal of Infectious Diseases. 2005; 191:S1-S6. [PubMed: 15627219]

ArcGIS Desktop. Release 10 ed. Redlands, CA, USA: Environmental Systems Research Institute; 2011.

Badland H, Schofield G, Witten K, Schluter P, Mavoa S, Kearns R, Hinckson E, Oliver M, Kaiwai H, Jensen V, Ergler C, McGrath L, McPhee J. Understanding the Relationship between Activity and Neighbourhoods (URBAN) Study: research design and methodology. BMC Public Health. 2009; 9:224. [PubMed: 19589175]

Blanchard JF, Aral SO. Emergent properties and structural patterns in sexually transmitted infection and HIV research. Sexually Transmitted Infections. 2010; 86:iii4-iii9. [PubMed: 21098056]

Bourgois P, Prince B, Moss A. The Everyday Violence of Hepatitis C Among Young Women Who Inject Drugs in San Francisco. Hum Organ. 2004; 63:253-264. [PubMed: 16685288]

City of Vancouver Open Data Catalogue. [Retrieved January 1 2012] from http://data.vancouver.ca/ datacatalogue/index.htm.

Cohen D, Spear S, Scribner R, Kissinger P, Mason K, Wildgen J. "Broken windows" and the risk of gonorrhea. Am J Public Health. 2000; 90:230-236. [PubMed: 10667184]

Cooper H, Des Jarlais D, Ross Z, Tempalski B, Bossak B, Friedman S. Spatial Access to Sterile Syringes and the Odds of Injecting with an Unsterile Syringe among Injectors: A Longitudinal Multilevel Study. Journal of Urban Health. 2012a; 89:678-696. [PubMed: 22585448]

Cooper HLF, Des Jarlais DC, Tempalski B, Bossak BH, Ross Z, Friedman SR. Drug-related arrest rates and spatial access to syringe exchange programs in New York City health districts: Combined effects on the risk of injection-related infections among injectors. Health \&amp; Place. 2012b; 18:218-228. [PubMed: 22047790]

Deering KN, Kerr T, Tyndall MW, Montaner JSG, Gibson K, Irons L, Shannon K. A peer-led mobile outreach program and increased utilization of detoxification and residential drug treatment among female sex workers who use drugs in a Canadian setting. Drug and Alcohol Dependence. 2011; 113:46-54. [PubMed: 20727683]

Diez Roux AV, Aiello AE. Multilevel Analysis of Infectious Diseases. Journal of Infectious Diseases. 2005; 191:S25-S33. [PubMed: 15627228]

DMTI Spatial Data and Services. [Retrieved January 31 2012] from http://www.dmtispatial.com/.

Fairbairn N, Small W, Shannon K, Wood E, Kerr T. Seeking refuge from violence in street-based drug scenes: Women's experiences in North America's first supervised injection facility. Social Science \& Medicine. 2008; 67:817-823. [PubMed: 18562065]

Ghose T, Swendeman DT, George SM. The Role of Brothels in Reducing HIV Risk in Sonagachi, India. Qual Health Res. 2011; 21:587-600. [PubMed: 21266706] 
Hubbard P. Community action and the displacement of street prostitution: evidence from British cities. Geoforum. 1998; 29:269-286.

Hubbard P, Whowell M. Revisiting the red light district: Still neglected, immoral and marginal? Geoforum. 2008; 39:1743-1755.

Janssen PA, Gibson K, Bowen R, Spittal PM, Petersen KL. Peer support using a mobile access van promotes safety and harm reduction strategies among sex trade workers in Vancouver's downtown eastside. Journal of Urban Health. 2009; 86:804-809. [PubMed: 19533367]

Kerr T, Wood E. The public health and social impacts of drug market enforcement: A review of the evidence. Int J Drug Pol. 2005; 16:210-220.

Kerrigan D, Ellen JM, Moreno L, Rosario S, Katz J, Celentano DD, Sweat M. Environmentalstructural factors significantly associated with consistent condom use among female sex workers in the Dominican Republic. AIDS. 2003; 17:415-423. [PubMed: 12556696]

Kroeger GL, Messer L, Edwards SE, Miranda ML. A novel tool for assessing and summarizing the built environment. Int J Health Geogr. 2012; 11:46. [PubMed: 23075269]

Krusi A, Chettiar J, Ridgway A, Abbott J, Strathdee SA, Shannon K. Negotiating Safety and Sexual Risk Reduction With Clients in Unsanctioned Safer Indoor Sex Work Environments: A Qualitative Study. American Journal of Public Health. 2012; 102:1154-1159. [PubMed: 22571708]

Kwiatkowski C, Booth R. Differences in HIV Risk Behaviors Among Women Who Exchange Sex for Drugs, Money, or Both Drugs and Money. AIDS and Behavior. 2000; 4:233-240.

Lazarus L, Chettiar J, Deering K, Nabess R, Shannon K. Risky health environments: Women sex workers' struggles to find safe, secure and non-exploitative housing in Canada's poorest postal code. Social Science \& Medicine. 2011; 73:1600-1607. [PubMed: 22018526]

Lima VD, Harrigan R, Murray M, Moore DM, Wood E, Hogg RS, Montaner JS. Differential impact of adherence on long-term treatment response among naive HIV-infected individuals. AIDS. 2008; 22:2371-2380. 2310.1097/QAD.2370b2013e328315cdd328313. [PubMed: 18981777]

Lowman J. Street prostitution control: some Canadian reflections on the Finsbury Park experience. British Journal of Criminology. 1992; 32

Lowman J. Violence and the Outlaw Status of (Street) Prostitution in Canada. Violence Against Women. 2000; 6:987-1011.

Maher, L. Sexed work: Gender, race and resistance in a Brooklyn drug market. New York: Oxford University Press; 1997.

Maher L, Curtis R. Women on the edge of crime: Crack cocaine and the changing contexts of streetlevel sex work in New York City. Crime, Law and Social Change. 1992; 18:221-258.

Maher L, Dunlap E, Johnson BD, Hamid A. Gender, power, and alternative living arrangements in the inner-city crack culture. Journal of Research in Crime and Delinquency. 1996; 33:181-205.

Maldonado G, Greenland S. Simulation Study of Confounder-Selection Strategies. American Journal of Epidemiology. 1993; 138:923-936. [PubMed: 8256780]

McGinn AP, Evenson KR, Herring AH, Huston SL, Rodriguez DA. Exploring Associations between Physical Activity and Perceived and Objective Measures of the Built Environment. J Urban Health. 2007; 84:162-184. [PubMed: 17273926]

Nosyk B, Wood E. Evidence-based drug policy: It starts with good evidence and ends with policy reform. The International journal on drug policy. 2012; 23:423-425. [PubMed: 23159128]

Rhodes T. The 'risk environment': a framework for understanding and reducing drug-related harm. International Journal of Drug Policy. 2002; 13:85-94.

Rhodes T. Risk environments and drug harms: a social science for harm reduction approach. Int J Drug Policy. 2009; 20:193-201. [PubMed: 19147339]

Rhodes T, Simic M, Baros S, Platt L, Zikic B. Police violence and sexual risk among female and transvestite sex workers in Serbia: qualitative study. BMJ. 2008; Vol. 337:a811. [PubMed: 18667468]

Rhodes, T.; Wagner, K.; Strathdee, S.; Shannon, K.; Davidson, P.; Bourgois, P. Structural Violence and Structural Vulnerability Within the Risk Environment: Theoretical and Methodological Perspectives for a Social Epidemiology of HIV Risk Among Injection Drug Users and Sex Workers. In: O'Campo, P.; Dunn, JR., editors. Rethinking Social Epidemiology. Netherlands: Springer; 2012. p. 205-230. 
Rusch MLA, Brouwer KC, Lozada R, Strathdee SA, Magis-RodrÃguez C, Patterson TL. Distribution of Sexually Transmitted Diseases and Risk Factors by Work Locations Among Female Sex Workers in Tijuana, Mexico. Sexually Transmitted Diseases. 2010; 37:608-614. [PubMed: 20585278]

SAS Version 9.3. Cary, USA: SAS Institute Inc; 2012.

Shahmanesh M, Patel V, Mabey D, Cowan F. Effectiveness of interventions for the prevention of HIV and other sexually transmitted infections in female sex workers in resource poor setting: a systematic review. Tropical Medicine \& International Health. 2008; 13:659-679. [PubMed: 18266784]

Shannon K. The hypocrisy of Canada's prostitution legislation. CMAJ. 2010; 182:1388. [PubMed: 20713573]

Shannon K, Bright V, Parsad D, Alexson D, Allinott S, Gibson K, Tyndall MW. Community-Based HIV prevention research among substance-using women in survival sex work: The Maka Project. Harm Reduction Journal. 2007:20-26. [PubMed: 18067670]

Shannon K, Csete J. Violence, Condom Negotiation, and HIV/STI Risk Among Sex Workers. JAMA: The Journal of the American Medical Association. 2010; 304:573-574. [PubMed: 20682941]

Shannon K, Kerr T, Allinott S, Chettiar J, Shoveller J, Tyndall MW. Social and structural violence and power relations in mitigating HIV risk of drug-using women in survival sex work. Social Science and Medicine. 2008a; 66:911-921. [PubMed: 18155336]

Shannon K, Kerr T, Bright V, Gibson K, Tyndall MW. Drug sharing with clients as a risk marker for increased violence and sexual and drug-related harms among survival sex workers. AIDS Care. 2008b; 20:235-241. [PubMed: 18293135]

Shannon K, Kerr T, Strathdee SA, Shoveller J, Montaner JS, Tyndall MW. Prevalence and structural correlates of gender based violence among a prospective cohort of female sex workers. BMJ. 2009; 339:b2939. [PubMed: 19671935]

Shannon K, Rusch M, Shoveller J, Alexson D, Gibson K, Tyndall MW. Mapping violence and policing as an environmental-structural barrier to health service and syringe availability among substance-using women in street-level sex work. Int J Drug Policy. 2008c; 19:140-147. [PubMed: 18207725]

Small W, Kerr T, Charette J, Schechter MT, Spittal PM. Impacts of intensified police activity on injection drug users: Evidence from an ethnographic investigation. International Journal of Drug Policy. 2006; 17:85-95.

Stueve A, O'Donnell LN, Duran R, San Doval A, Blome J. Time-Space Sampling in Minority Communities: Results With Young Latino Men Who Have Sex With Men. Am J Public Health. 2001; 91:922-926. [PubMed: 11392935]

Tempalski B, McQuie H. Drugscapes and the role of place and space in injection drug use-related HIV risk environments. The International journal on drug policy. 2009; 20:4-13. [PubMed: 18554896]

Withers M, Dornig K, Morisky DE. Predictors of workplace sexual health policy at sex work establishments in the Philippines. AIDS Care. 2007; 19:1020-1025. [PubMed: 17851999]

Wood E, McKinnon M, Strang R, Kendall PR. Improving community health and safety in Canada through evidence-based policies on illegal drugs. Open Medicine. 2012; 6:e35-e40. [PubMed: 22567081] 


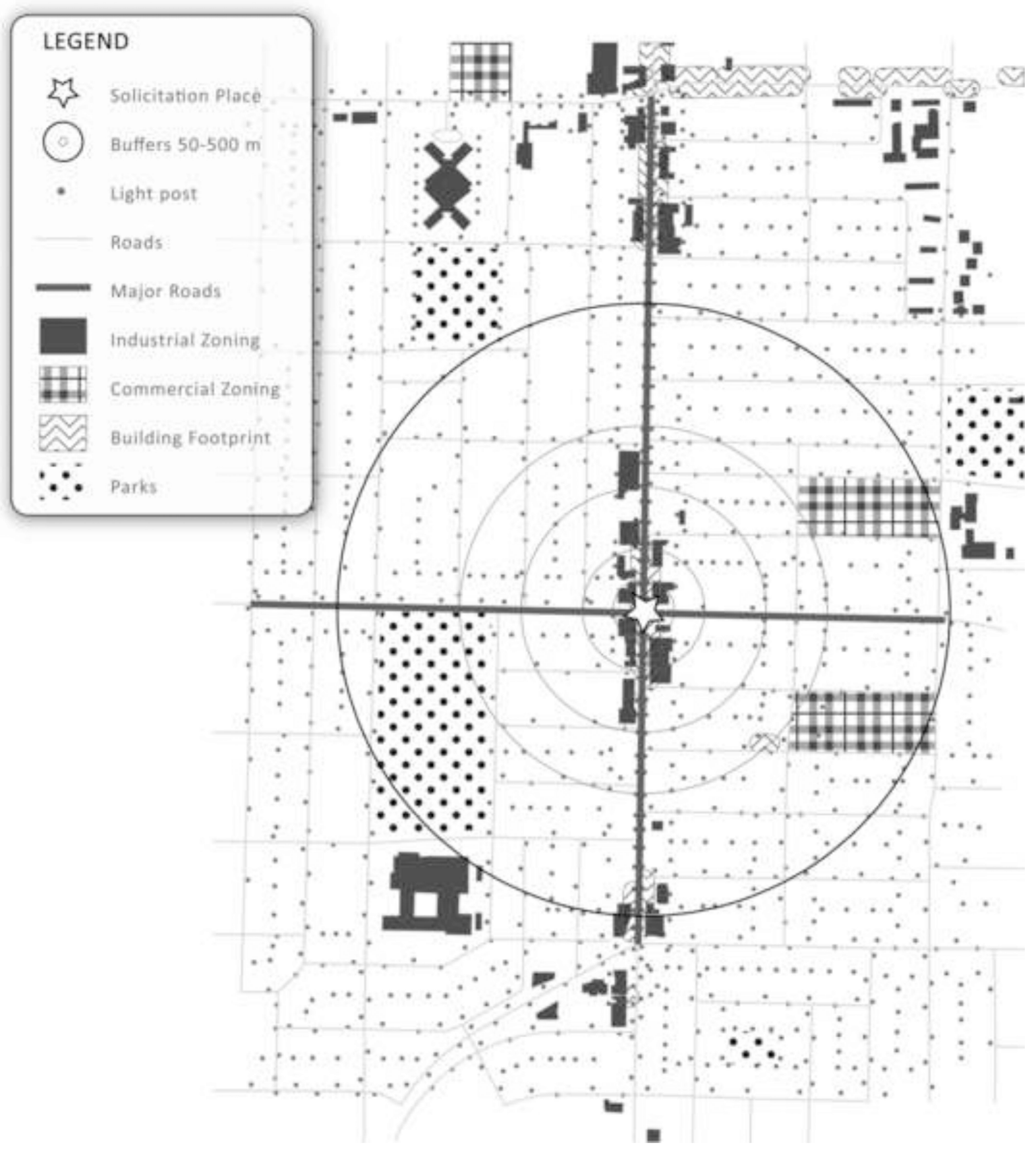

Figure 1.

Representation of how built environment indicators were derived (not an actual map of Vancouver); black dot represents sex workers' main place of solicitation or servicing clients, with black circles representing different potential buffer sizes (the current study used 50m buffers); green squares represent parks; red squares represent commercial zoning; light green areas represent industrial zoning; yellow areas represent building footprint; red dots 
represent light posts. Descriptions of seven derived built environment indicators are in Table 1 . 
Table 1

Indicators of the built environment, developed for a buffer size of 50m

\begin{tabular}{llll}
\hline Group & Description & Variable & Source \\
\hline Roads & Sum of road length within a buffer & Major Roads & DMTI \\
\cline { 3 - 4 } & & Alley Roads & City of Vancouver \\
\hline Land Use & \% of land use of total buffer area & Commercial & DMTI \\
\cline { 3 - 4 } & & Industrial & DMTI \\
\cline { 2 - 3 } & & Parks & DMTI \\
\hline Lighting & Number of light posts within a buffer & Lighting & City of Vancouver \\
\hline Building Footprint & $\%$ of building footprint of total buffer area & Building footprint & City of Vancouver \\
\hline
\end{tabular}




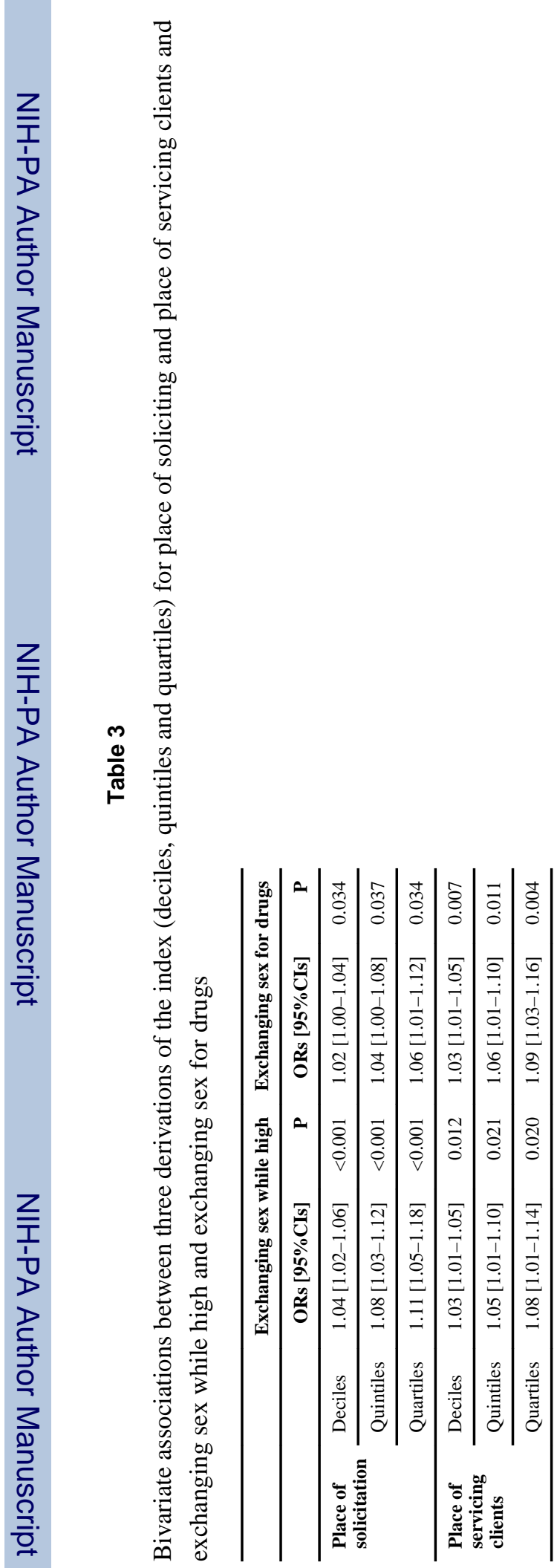




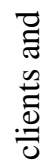

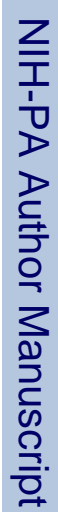

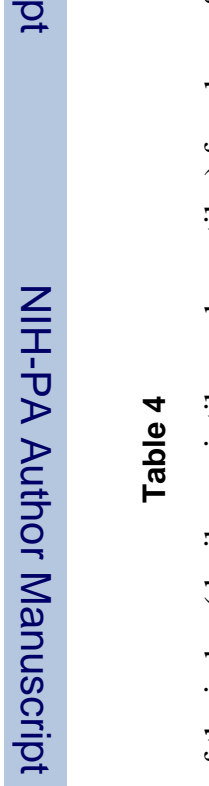

Z

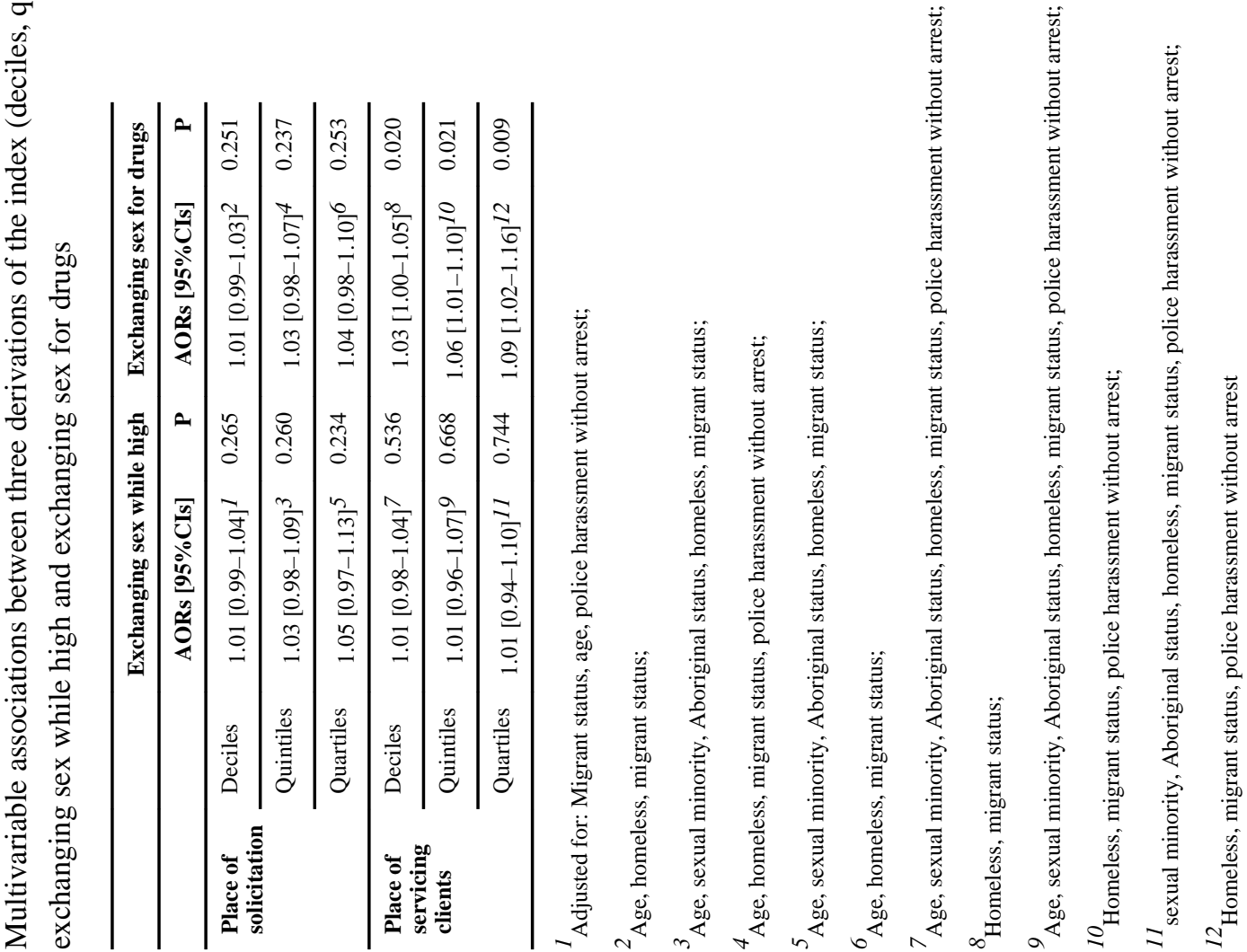




\section{Table 5}

(a) Multivariable associations between each built environment indicator (place of solicitation) and exchanging sex while high and exchanging sex for drugs in the last six months; (b) Multivariable associations between each individual built environment indicator and exchanging sex while high and exchanging sex for drugs, adjusting for police harassment

\begin{tabular}{|c|c|c|c|c|}
\hline \multirow[t]{2}{*}{ Environmental/land use variable } & \multicolumn{2}{|c|}{ Exchanged sex while high } & \multicolumn{2}{|c|}{ Exchanged sex for drugs } \\
\hline & AOR $[95 \% \mathrm{CIs}]$ & $\mathbf{P}$ & AOR $[95 \%$ CIs] & $\mathbf{P}$ \\
\hline Length of major roads within $50 \mathrm{~m}$ buffer & $1.05[0.77-1.45]^{1}$ & 0.751 & $0.89[0.69-1.14]^{2}$ & 0.346 \\
\hline Length of alley roads within $50 \mathrm{~m}$ buffer & $\mathrm{ns}$ & & $\mathrm{ns}$ & \\
\hline$\%$ Commercial areas within 50m buffer & $0.76[0.59-0.99]^{3}$ & 0.044 & $\mathrm{~ns}$ & \\
\hline$\%$ Industrial areas within $50 \mathrm{~m}$ buffer & $1.14[0.83-1.56]^{4}$ & 0.421 & $\mathrm{~ns}$ & \\
\hline$\%$ of Parks within 50m buffer & $1.18[0.82-1.70]^{5}$ & 0.387 & $1.36[1.06-1.75]^{6}$ & 0.017 \\
\hline Count of lighting within $50 \mathrm{~m}$ buffer & $\mathrm{ns}$ & & $\mathrm{ns}$ & \\
\hline \% Built environment within 50m buffer & $\mathrm{ns}$ & & $\mathrm{ns}$ & \\
\hline
\end{tabular}

(b)

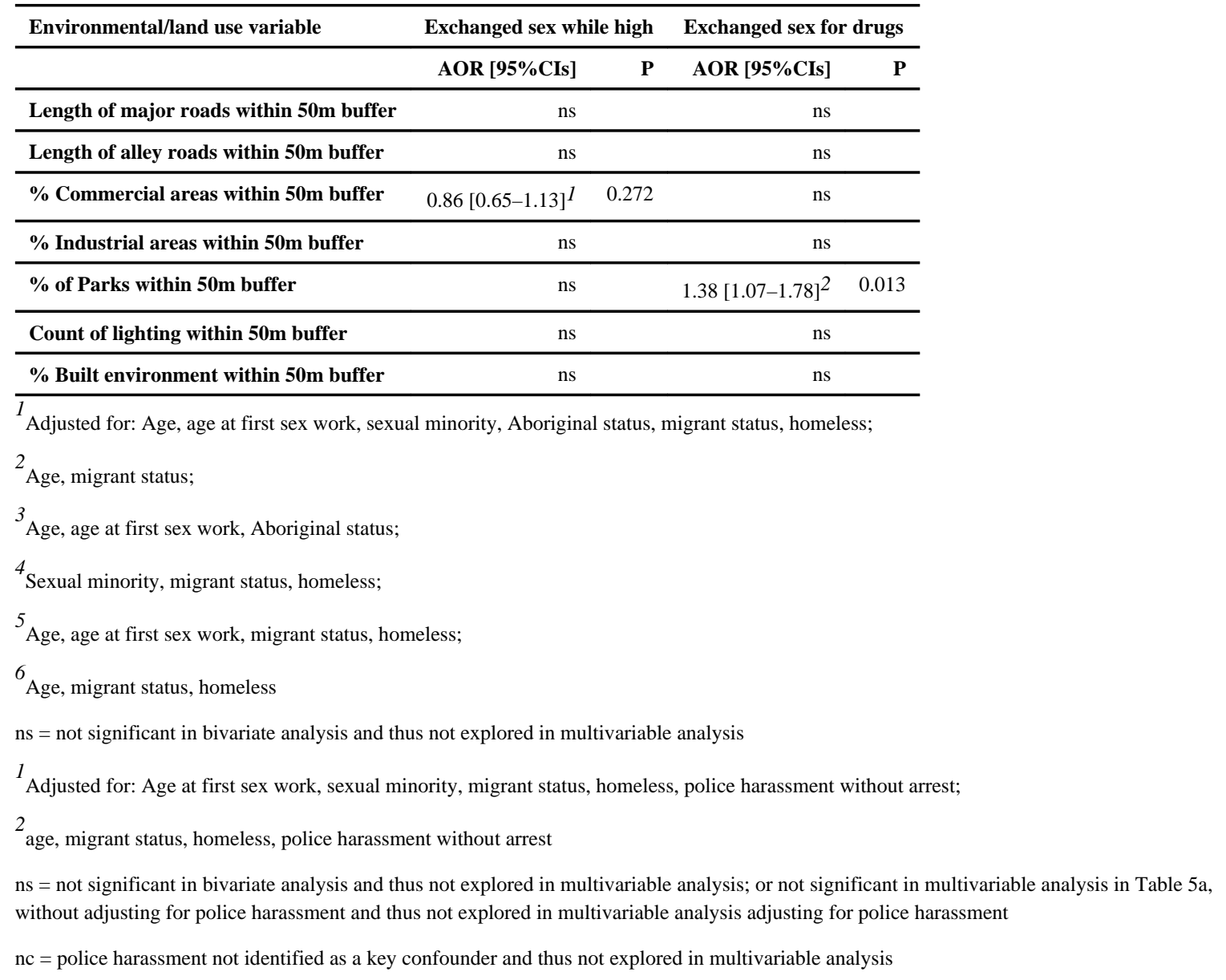




\section{Table 6}

(a) Multivariable associations between each built environment indicator (place of servicing) and exchanging sex while high and exchanging sex for drugs in the last six months; (b) Multivariable associations between each individual built environment indicator and exchanging sex while high and exchanging sex for drugs, adjusting for police harassment

\begin{tabular}{lrrrrr} 
(a) & \multicolumn{1}{c}{ Exchanged sex while high } & \multicolumn{2}{c}{ Exchanged sex for drugs } \\
\hline Lenvironmental/land use variable & AOR [95\% CIs] & P & AOR [95\% CIs] & P \\
\hline Length of alley roads within 50m buffer & $0.69[0.53-0.89]^{2}$ & 0.005 & & $0.84[0.66-1.06]^{1}$ & 0.144 \\
\hline \% Commercial areas within 50m buffer & $0.82[0.64-1.06]^{3}$ & 0.130 & $0.72[0.57-0.92]^{4}$ & 0.007 \\
\hline \% Industrial areas within 50m buffer & $1.31[1.00-1.71]^{5}$ & 0.051 & & \\
\hline \% of Parks within 50m buffer & $1.08[0.77-1.51]^{6}$ & 0.662 & $1.58[1.14-2.18]^{7}$ & 0.006 \\
\hline Count of lighting within 50m buffer & & $\mathrm{ns}$ & $0.73[0.56-0.93]^{8}$ & 0.012 \\
\hline \% Built environment within 50m buffer & & $\mathrm{ns}$ & $0.75[0.60-0.95]^{9}$ & 0.017 \\
\hline
\end{tabular}

(b)

\begin{tabular}{|c|c|c|c|c|}
\hline \multirow[t]{2}{*}{ Environmental/land use variable } & \multicolumn{2}{|c|}{ Exchanged sex while high } & \multicolumn{2}{|c|}{ Exchanged sex for drugs } \\
\hline & AOR $[95 \% \mathrm{CIs}]$ & $\mathbf{P}$ & AOR $[95 \% \mathrm{CIs}]$ & $\mathbf{P}$ \\
\hline Length of major roads within $50 \mathrm{~m}$ buffer & $\mathrm{ns}$ & & $\mathrm{ns}$ & \\
\hline Length of alley roads within $50 \mathrm{~m}$ buffer & $\mathrm{nc}$ & & $\mathrm{ns}$ & \\
\hline$\%$ Commercial areas within 50m buffer & ns & & $0.74[0.58-0.93]^{l}$ & 0.012 \\
\hline$\%$ Industrial areas within $50 \mathrm{~m}$ buffer & $\mathrm{ns}$ & & ns & \\
\hline$\%$ of Parks within 50m buffer & ns & & $\mathrm{nc}$ & \\
\hline Count of lighting within $50 \mathrm{~m}$ buffer & $\mathrm{ns}$ & & $\mathrm{nc}$ & \\
\hline$\%$ Built environment within 50m buffer & $\mathrm{ns}$ & & $0.76[0.60-0.97]^{2}$ & 0.025 \\
\hline \multicolumn{5}{|l|}{ Adjusted for: Age, migrant status, homeless; } \\
\hline \multicolumn{5}{|l|}{2 Migrant status, homeless; } \\
\hline \multicolumn{5}{|c|}{${ }^{3}$ Age, sexual minority, Aboriginal status, homeless; } \\
\hline \multicolumn{5}{|c|}{${ }^{4}$ Migrant status, homeless; } \\
\hline \multicolumn{5}{|l|}{5 Aboriginal status, homeless; } \\
\hline \multicolumn{5}{|c|}{${ }^{6}$ Age, age at first sex work, Aboriginal status, migrant status, homeless; } \\
\hline \multicolumn{5}{|c|}{7 Migrant status; } \\
\hline \multicolumn{5}{|l|}{${ }^{8}$ Migrant status; } \\
\hline \multicolumn{5}{|l|}{${ }^{9}$ Migrant status, homeless } \\
\hline
\end{tabular}


${ }^{2}$ Migrant status, homeless, police harassment without arrest

$\mathrm{ns}=$ not significant in bivariate analysis and thus not explored in multivariable analysis; or not significant in multivariable analysis in Table 5a, without adjusting for police harassment and thus not explored in multivariable analysis adjusting for police harassment

$\mathrm{nc}=$ police harassment not identified as a key confounder and thus not explored in multivariable analysis 